\title{
Crescent Shape MIMO Monopole Antenna for Wi-Fi/Wi-MAX Application
}

\author{
Sachin S. Khade ${ }^{1}$, S.L. Badjate ${ }^{2}$ \\ Assistant Professor, Yashwantrao Chavan College of Engg, Nagpur, India ${ }^{1}$ \\ Professor, S.B.J.I.T.M.R., Nagpur, India ${ }^{2}$
}

\begin{abstract}
This paper present a compact MIMO antenna for Wi-Fi/WiMAX application. The antenna consists of two crescents shaped Radiators placed symmetrically along the ground and are connected by the neutralization line to achieve good impedance matching and low mutual coupling. The compact antenna with a size of $40 \mathrm{~mm} \times 90 \mathrm{~mm}$ is proposed. The antenna works in two operating i.e. lower band range from $3.01 \mathrm{GHz}$ to $3.55 \mathrm{GHz}$ while upper band range from $5.55 \mathrm{GHz}$ to $6.54 \mathrm{GHz}$. The reflection coefficient in both band is achieved below $-10 \mathrm{~dB}$. Besides, port isolation, envelope correlation coefficient and radiation characteristics are also investigated.
\end{abstract}

Keywords: Mutual Coupling, Isolation, Gain, Multiple-Input Multiple Output (MIMO) Antenna, Radiation Pattern, Correlation Coefficient.

\section{INTRODUCTION}

During the last few years, the growing demand for highspeed wireless data access has promoted the development of broadband wireless access techniques such as Wireless fidelity (Wi-Fi) and World interoperability for microwave access (WiMAX). Multiple-input multiple-output (MIMO) technology implements multiple antenna elements on the transmitting and receiving ends of the MIMO system to achieve maximum channel capacity for delivering high data rate traffic even in a scattering environment. Elements with compact size and wideband characteristics have been proposed, and low-mutual coupling is achieved with various methods, like parasitic element. Theoretical and experimental published research articles [3], [4] confirm the data rate enhancement, multipath fading reduction and co-channel interference suppression capabilities [1], [2]when deploying MIMO technology.

Single element antenna is easy to implement but provide low data rate. In order to improve data rate MIMO antenna techniques are used. But in MIMO technique the small spacing between antenna elements introduces mutual coupling effect, which affect various parameters such as overall gain of antenna, isolation etc. Significant efforts is required, to reduce the mutual coupling or achieve good isolation between closely placed antenna elements have been made [2]. The neutralization technique is implemented by physically connecting the multiple antenna elements with a transmission line. All these methods offers narrow band isolation and very few of these offers wideband isolation. This paper introduces a isolation technique with two closely spaced monopole antennas. A Zigzag pattern neutralization line is used to connect two antennas together to improve isolation and reduces mutual coupling between the antenna elements.

\section{ANTENNA DESIGN AND SIMULATION}

\section{A. MIMO Antenna without Neutralization Line}

The proposed MIMO antenna with crescent shape is printed on the FR4 substrate having thickness of $1.6 \mathrm{~mm}$. Figure 1 shows the front and the bottom view of the antenna. On bottom side a defect is provided by inserting rectangular slit in ground plane. The radiator is fed by a $14 \times 1 \mathrm{~mm}$ Microstrip line which improves impedance matching. The radiators are printed on the upper side of FR4 substrate. The total size of the antenna is $40 \mathrm{~mm} \times 90$ $\mathrm{mm} \times 1.6 \mathrm{~mm}$.

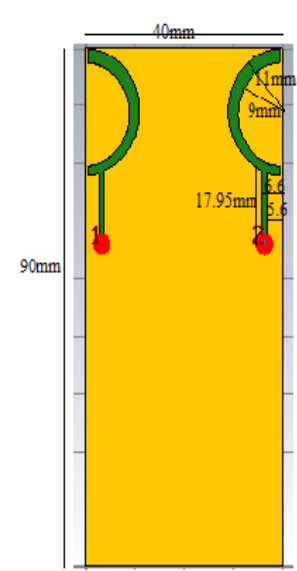

(a)Front view

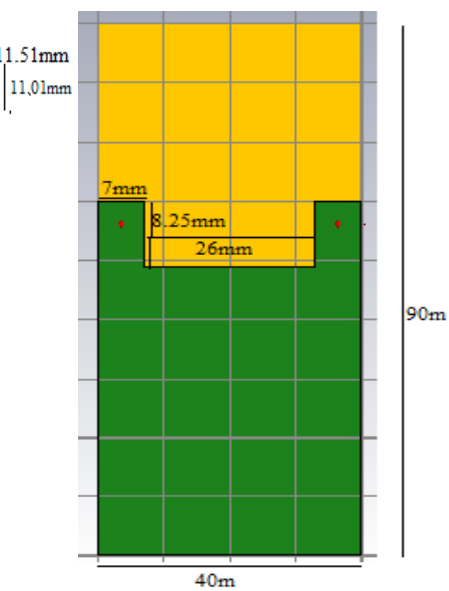

(b) Bottom View
Figure 1: Geometry of the proposed antenna

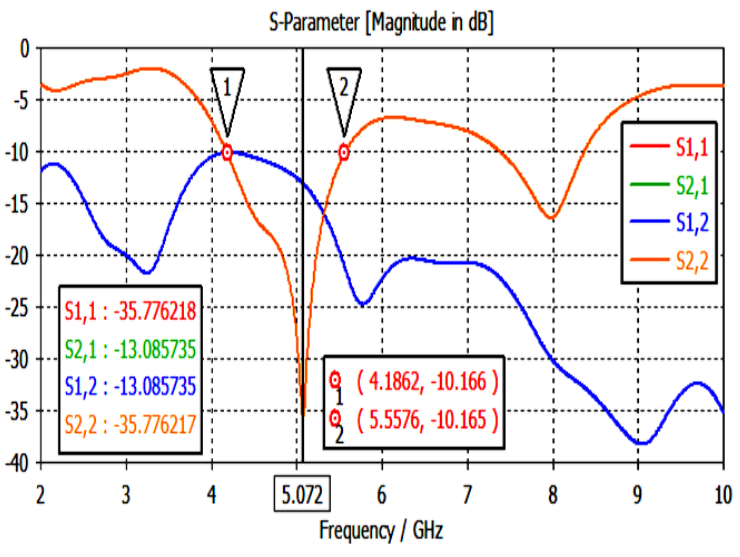

Figure.2 Simulated S-parameter 
After simulation, the two frequency bands are observed, radiators are separated by $20 \mathrm{~mm}$. In general, the one from 4.18-5.55 GHz with resonant frequency neutralization lines transfer some currents from the first $5.07 \mathrm{GHz}$ and other from $7.18-8.45 \mathrm{GHz}$ with resonant antenna and re-inject it in the second antenna with a frequency $7.95 \mathrm{GHz}$. the lower band is useful for WLAN suitable magnitude and phase to cancel out the existing and W-Max. The reflection coefficients (S11\&S22) observed at $5.07 \mathrm{GHz}$ is at $-35.77 \mathrm{~dB}$ and with isolation up to $-13.08 \mathrm{~dB}$ as shown in Figure 2.

\section{B. MIMO Antenna with slots in the Crescent}

As the isolation between elements is less and need to improve. So, to improve the mutual coupling by reducing spurious radiation, a Circular cutting slot of different radius are introduced into the radiators. These Slots are used to reduce the coupling effect caused by ground surface. The antenna with slots in the radiators is as shown in the Figure3.

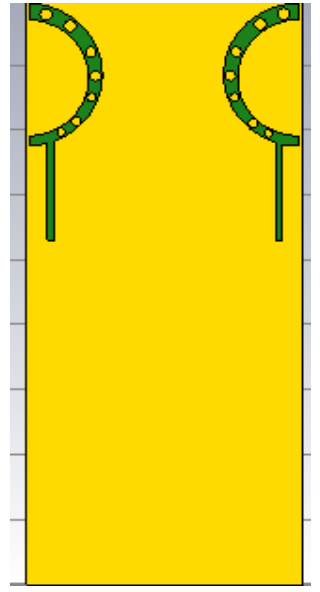

Figure3.Geometry of antenna with slots

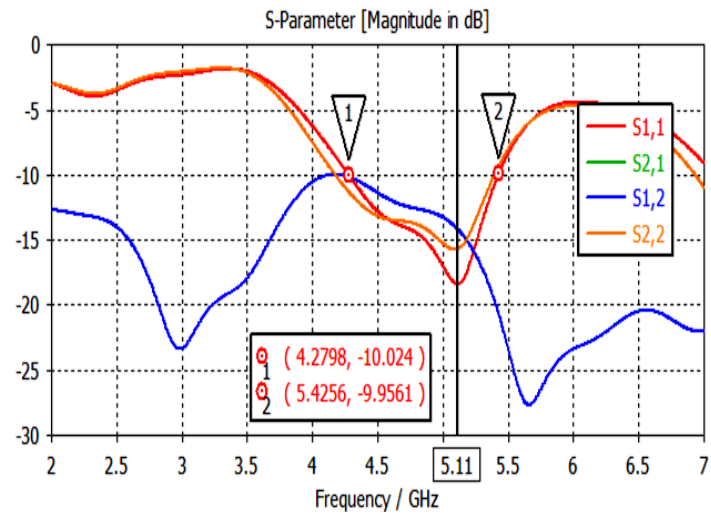

Figure4. Simulated S-Parameter

Due to insertion of Circular slots frequency band achieved by antenna is range from 4.27 to $5.42 \mathrm{GHz}$ with the resonant frequency of $5.11 \mathrm{GHz}$. The reflection coefficient $(\mathrm{S} 11, \mathrm{~S} 22)$ is observed at $5.11 \mathrm{GHz}$ is $-18.37 \mathrm{~dB}$ is shown in Fig.4. we got the value transmission coefficient (S12 \& $\mathrm{S} 21)$ below $-14 \mathrm{~dB}$, which indicate the isolation is improved. The VSWR for both elements are found around 1.27 at resonance frequency $5.11 \mathrm{GHz}$.

\section{MIMO Antenna with Neutralization Line}

To achieve sufficient isolation between the two identical elements and to maintain a good impedance matching over the desired frequency band, a new neutralization lines structure is introduced between the two radiators. The coupling current between two antenna elements. This current path contributes to enhance isolation at the highest edge of the frequency band. The additional network does not remove the inherent coupling between the antennas, but enhances the inter port isolation. The coupling network does not have attributes of a filter or resonator, and most resembles a branch line coupler introducing several paths between its nodes. The antenna with neutralization lines is as shown in the Figure 5.

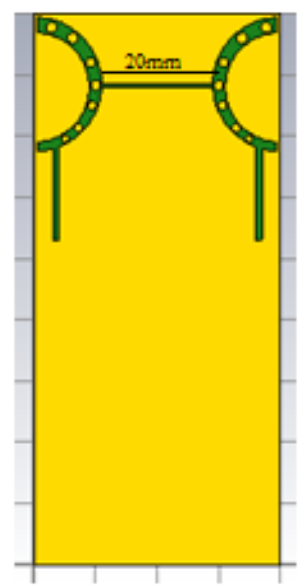

Figure5. Geometry of antenna with neutralization

The Neutralization line shifts the operating frequency band in between 4.41 to $5.81 \mathrm{GHz}$ with the resonant frequency at $5.31 \mathrm{GHz}$. The reflection coefficients (S11\& S22) are down up to $-21.94 \mathrm{~dB}$ at resonance frequency $5.31 \mathrm{GHz}$ as shown in Fig.6. But the isolation between elements is spoiled. The VSWR is found 1.17. While the gain of antenna is increased up to $4.22 \mathrm{~dB}$.

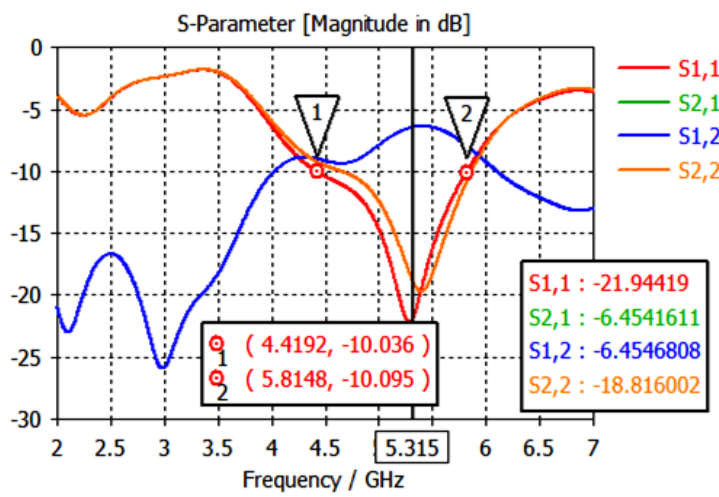

Figure6. Simulated S-parameters

\section{MIMO Antenna with Zigzag pattern Neutralization Line}

The simple neutralization line inserting between patch does not give isolation up to the mark. so to improve isolation additional structure is inserted. A neutralization line with zigzag pattern is introduced to achieve sufficient bandwidth and good impedance matching. The design of the proposed antenna with zigzag pattern neutralization line is as shown in the figure 7. 
Resonant modes can be established by the simulation characteristics of the proposed single element antenna obtained by manipulating the circles which constitutes of the radiators. When these two suitable modes are constructively merged, the desired impedance bandwidth can be obtained. Neutralization line is introduced between the two radiators to achieve sufficient isolation between the two identical elements to maintain a good impedance matching over the desired frequency band. Its function is to eliminate the original coupling current by providing an anti-phase coupling current. Hence, the coupling between two antenna elements can be weakened. Some currents from the first antenna is transferred due to the neutralization line and is injected in the second antenna with a suitable magnitude and phase to cancel out the coupling current between two antenna elements. The zigzag pattern neutralization line enhances port isolation.

With insertion of zigzag pattern neutralization line, two frequency band are observed as shown in figure 8 . The lower frequency band range in between frequencies 3.07 $\mathrm{GHz}$ to $3.55 \mathrm{GHz}$ with resonance frequency of $3.28 \mathrm{GHz}$, while upper frequency band range from $5.55 \mathrm{GHz}$ to 6.54 $\mathrm{GHz}$ with resonance frequency of $5.92 \mathrm{GHz}$. The reflection coefficient (S11 and S22) are observed below $22 \mathrm{~dB}$ at lower band and $-27 \mathrm{~dB}$ at upper band respectively. While the transmission coefficient (S12 and S21) are found around $-10 \mathrm{db}$ for lower ban and $-15 \mathrm{~dB}$ for upper band. Now this antenna structure become multiband Antenna with two operating bands.

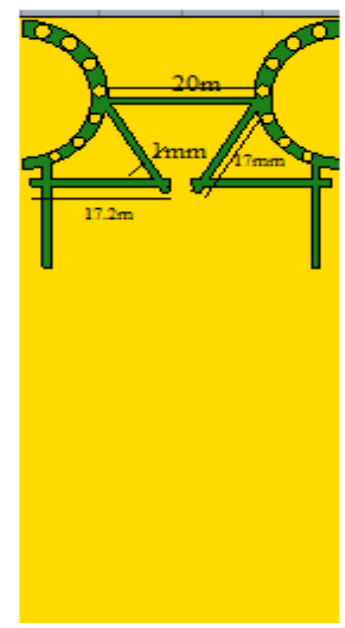

Figure7. Geometry of antenna with zigzag pattern neutralization line

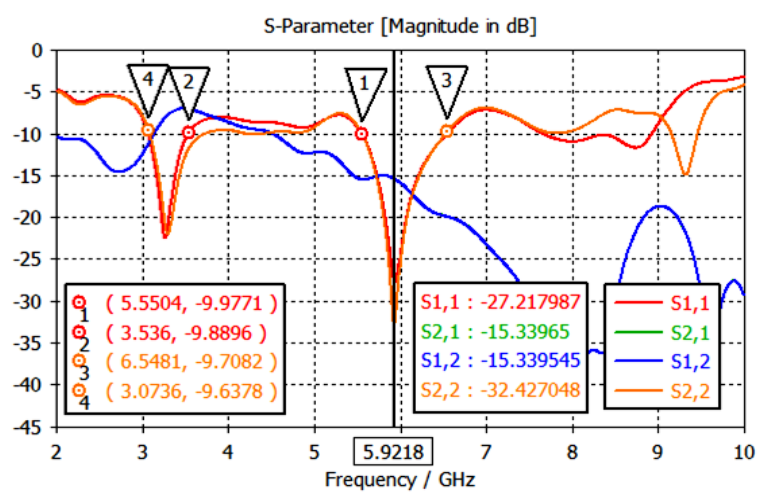

Figure8. Simulated S-parameters

\section{RESULTS AND DISCUSSION}

The simulated results are plotted in Fig. 8 to 12 . As we can be seen, the impedance bandwidth of the antenna encompasses the operating frequency spectra from 3.07 $\mathrm{GHz}$ to $3.55 \mathrm{GHz}$ for a reflection coefficient below $-10 \mathrm{~dB}$ with the centre frequency of $3.28 \mathrm{GHz}$, also from 5.55 $\mathrm{GHz}$ to $6.54 \mathrm{GHz}$ with centre frequency of $5.92 \mathrm{GHz}$. The bandwidth achieved in both band fully covers the requirements of WiMAX / Wi-Fi.

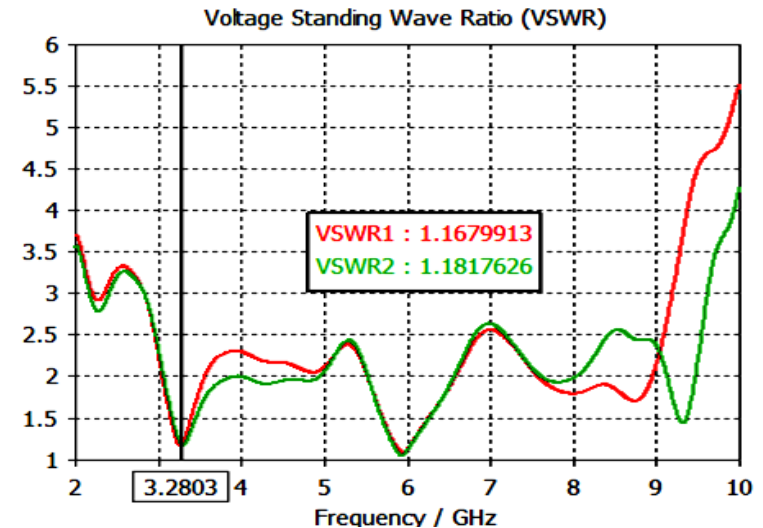

Fig. 9 (a) VSWR for frequency $3.28 \mathrm{GHz}$

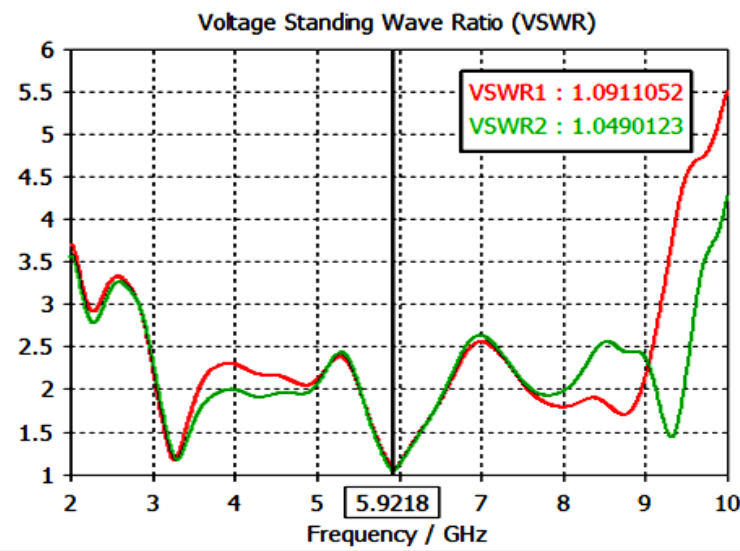

Fig. 9 (b) VSWR for frequency $5.92 \mathrm{GHz}$

The VSWR is about 1.18 at lower band and 1.09 at upper band as shown in figure 9 .

The 3D radiation pattern of antenna shown in figure 10 . The gain achieved by antenna with neutralization line good enough in both bands. In lower band the value of gain is found $3.72 \mathrm{dBi}$ with directivity of $4.01 \mathrm{dBi}$, while the gain at upper band is found $3.99 \mathrm{dBi}$ with directivity of $4.55 \mathrm{dBi}$.

The $2 \mathrm{D}$ radiation pattern of antennas is shown in figure 11 and 12. The vertical and horizontal far field shows the direction of propagation of the signal. For diversity and MIMO application, the correlation coefficient between the two antenna patterns is another paramount factor in the usefulness of this antenna, since it is directly connected with the loss of spectral efficiency and degradation of performance of a MIMO system. In general, the envelope correlation coefficient of an antenna array can be computed by using either the far-field radiation pattern or scattering parameters from the antenna system. 
The correlation coefficient between antenna elements is neutralization lines and a current of similar magnitude in found below 0.005.The maximum total radiation the top of the neutralization line is clearly seen to cancel efficiency achieve up to $85 \%$.
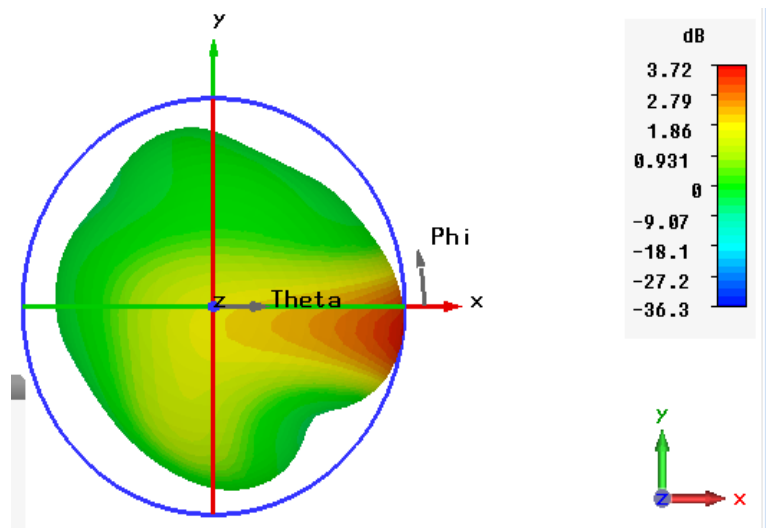

Figure 10 a) Gain at $3.28 \mathrm{GHz}$
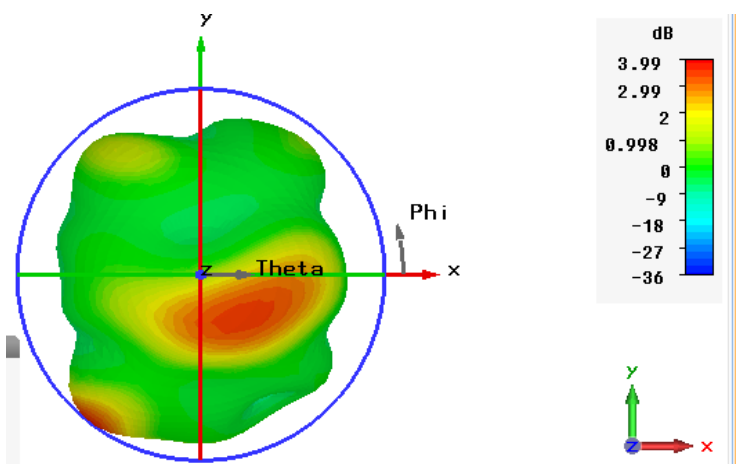

Figure 10 b) Gain at $5.92 \mathrm{GHz}$

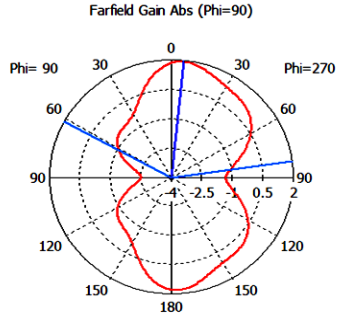

Theta / Degree vs. dB

(a) Vertical far field gain

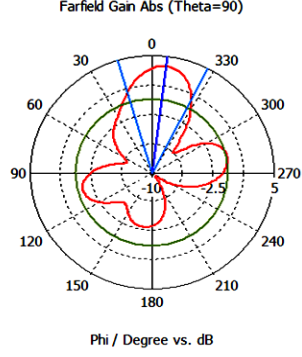

(b) Horizontal far field gain
Fig.11 2D gain at frequency $3.28 \mathrm{GHz}$

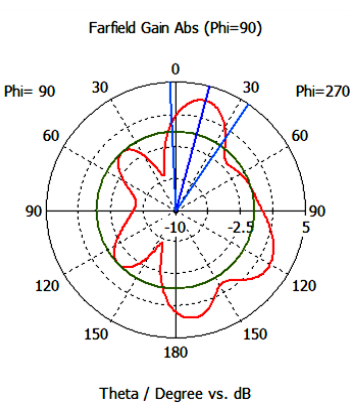

(a) Vertical far field gain

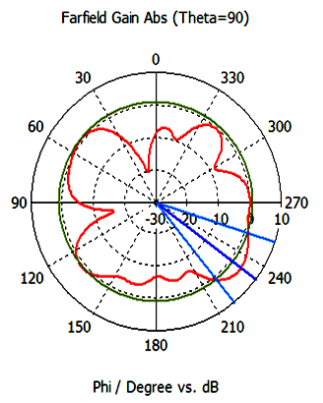

(b) Horizontal far field gain

Fig.12 2D gain at frequency $5.92 \mathrm{GHz}$

By inserting the lines, it can be noticed that the surface currents on both radiators are transferred to the this feed line current. This tends to decouple the currents on the port 2 radiator and hence it enhances the isolation between the two radiator elements efficiently.

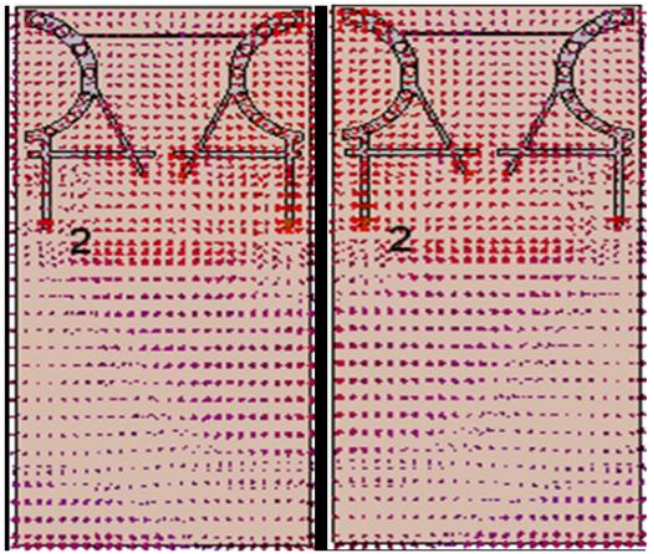

Figure 13 Current density

\section{CONCLUSION}

A compact MIMO crescent-shaped diversity monopole antenna with dimensions of $40 \mathrm{~mm} \times 90 \mathrm{~mm} \times 1.6 \mathrm{~mm}$ is proposed. By implementing the neutralization lines, the performance of MIMO antenna can be enhanced significantly over both frequency bands from $3.07 \mathrm{GHz}$ to $3.55 \mathrm{GHz}$, and $5.55 \mathrm{GHz}$ to $6.54 \mathrm{GHz}$ which would offer reliable wireless connectivity with a high data throughput in the expanding Wi-Fi/WiMAX service applications. The isolation of antenna is improved by inserting neutralization line between antenna elements. The maximum efficiency achieved by antenna is $85 \%$ with correlation coefficient below 0.005 .

\section{REFERENCES}

[1] Chan Hwang See, Raed A. Abd-Alhameed,Peter S. Excell,"Wideband Printed MIMO/Diversity Monopole Antenna for WiFi / WiMAX Applications "IEEE transactions on antennas and propagation, vol. 60, no. 4, april 2012.

[2] H. Shin and J. H. Lee, "Capacity of multiple-antenna fading channels:Spatial fading correlation, double scattering, and keyhole,"IEEETrans. Inform. Theory, vol. 49, no. 10, pp. 26362647, Oct. 2003.

[3] R. G. Vaughnan and J. B. Andersen, "Antenna diversity in mobilecommunication," IEEE Trans. Veh. Technol., vol. VT-36, no. 4, pp.149-172, 1987.

[4] D. Pozar, "Input impedance and mutual coupling of rectangular microstripantennas," IEEE Trans.Antennas Propag., vol. AP-30, pp.1191-1196, Nov. 1982.

[5] D. Ahn, J.-S. Park, C.-S. Kim, J. Kim, Y. Qian, and T. Itoh, "A designof the low-pass filter using the novel microstrip defected ground structure,"IEEE Trans. Microw. Theory Tech., vol. 49, no. 1, pp. 86-93,Jan. 2001

[6] Z. Li, K. Ito, Z. Du, and K. Gong, "Compact wideband printed diversityantenna for mobile handsets,"in Proc. Asia-Pac. Radio Sci. Conf.,Toyama, Japan, Oct. 2010, pp. 1-4.

[7] A. Diallo, C. Lxey, P. L. Thuc, R. Staraj, and G. Kossiavas, "Enhancedtwo-antenna structures for universal mobile telecommunicationssystem diversity terminals," IET Microw. Antennas Propag., vol.2, no. 1, pp. 93-101, Feb. 2008.

[8] A. Chebihi, C. Luxey, A. Diallo, P. L. Thuc, and R. Staraj, "A novel isolationtechnique for closely spaced PIFAs for UMTS mobile phones," IEEE Antennas Wireless Propag. Lett., vol. 7, pp. 665-668, 2008. 


\section{BIOGRAPHIES}

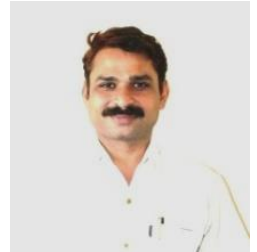

Sachin S. Khade (born December 31, 1976) is working as Assistant Professor in Department of Electronics and Telecommunication Engineering at Yashwantrao Chavan College of Engineering, Nagpur, India. He is passionately interested in simulation and modeling of electronic circuits, including RF circuits and Antenna. He has published more than fourteen research papers on a wide variety of topics in the domain of Antenna and microwaves. He has about six years of industry experience and more than ten years of academic experience. Simulation and modeling of $\mathrm{RF} /$ microwave components, sub-systems, and systems are one of his key passions. He is a Life Member of ISTE and ISRD. He has other technical membership in his credit like - IEEE, IACSIT, IAENG and UACEE.

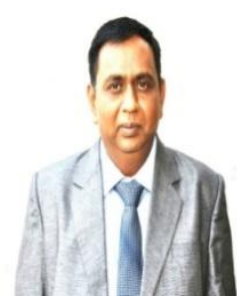

Sanjay L. Badjate is working as a Vice Principal at S.B. Jain institute of Technology Management and Research. He has total teaching experience 24 years, administrative experience of 15 years and 5 years in Research and Development.

$\mathrm{He}$ has got more than 65 research papers to his credit in international and national Journals and Conferences. He has co-authored various research papers on a wide variety of topics. He is a reviewer of many reputed international Journals and member programme committee of various National \& International Conferences. He has completed his B.E. from Nagpur University and M.E from Devi Ahilyabai University, Indore \& completed $\mathrm{Ph}$. D from Amravati University, Amravati. His areas of interest are Artificial Neural Network (ANN), soft computing; signal processing, RF/ Microwave \& Biomedical engineering etc. He is a Life member of ISTE \& Fellow member of IETE. 